\title{
Original
}

\section{On-Demand Inhaled Corticosteroid and Fast-Acting Beta-2 Agonist Combination Therapy Versus Regular Inhaled Corticosteroid Plus Rescue Bronchodilator in Adults with Mild Asthma : A Network Meta-Analysis}

\author{
Koichi Ando*1,2), Akihiko TANAKA ${ }^{1)}$, Takuya YokOE $^{1)}$, \\ Tsukasa OHNISHI ${ }^{1)}$, Shin INOUE $^{2)}$ and Hironori SAGARA ${ }^{1)}$
}

\begin{abstract}
The aim of this study was to assess the non-inferiority of on-demand (OD) inhaled corticosteroid (ICS) and fast-acting beta-2 agonist (FABA) combination therapy. Although regular inhalation of low-dose ICS and OD short-acting beta-2 agonist (REG-ICS + OD-SABA) is said to be effective therapy for mild asthma, we investigated whether OD-ICS / FABA is as effective as REG-ICS + ODSABA. A network meta-analysis of randomized controlled trials was conducted to examine non-inferiority by comparing the efficacy of NON-REG + OD-ICS / FABA with REG-ICS + OD-SABA for mild asthma. We also assessed the superiority of NON-REG + OD-ICS / FABA to OD-SABA without any regular treatment (NONREG + OD-SABA). PubMed, the Cochrane library database, and Scopus were searched to identify relevant articles with an outcome measure of the incidence of asthma control. A network meta-analysis was performed and the summary effect size was expressed as the mean difference (MD) with $95 \%$ confidence intervals (CIs). The probability of being the best treatment for the outcome and the surface under the cumulative ranking curves were also calculated. Three randomized controlled trials of treatment for mild asthma met the criteria and were included in the study. Non-inferiority of NON-REG + OD-ICS / FABA to REG-ICS + ODSABA (MD, $-0.17 ; 95 \% \mathrm{CI},-0.41$ to 0.07$)$ and superiority of NON-REG + ODICS / FABA to NON-REG + OD-SABA (MD, $-0.29 ; 95 \% \mathrm{CI},-0.49$ to -0.08$)$ were shown in patients with mild asthma. The respective probabilities of being the best treatment for asthma control were $91.2 \%, 8.8 \%$ and $0.2 \%$ for NON-REG + ODICS / FABA, REG-ICS + OD-SABA, and NON-REG + OD-SABA, respectively, and the surface under the cumulative ranking curves were 1.0, 0.5, and 0.1, respectively. These results suggest that NON-REG + OD-ICS / FABA is an effective alternative to REG-ICS + OD-SABA for asthma control in patients with mild asthma.
\end{abstract}

Key words : asthma, inhaled corticosteroid, beta-2 agonist, network meta-analysis

\footnotetext{
1) Department of Medicine, Division of Respiratory Medicine and Allergology, Showa University School of Medicine, 1-5-8 Hatanodai, Shinagawa-ku, Tokyo 142-8666, Japan.

2) Showa University Dental Hospital Medical Clinic.

* To whom corresponding should be addressed.
} 


\section{Introduction}

The frequency of patient hospitalization for acute exacerbation of bronchial asthma has decreased markedly in recent decades due to the global use of inhaled corticosteroids (ICS) ${ }^{1)}$. The 2014 revision of the Japanese Guidelines for Adult Asthma recommends treatment with regular ICS regardless of asthma severity because of the efficacy of ICS for the suppression of airway inflammation and inhibition of asthma progression ${ }^{2)}$. However, success in treating patients with asthma can be limited by poor adherence to daily inhaled medication ${ }^{3)}$. On-demand (OD) use may reduce the influence of poor adherence, and may lead to improved control of asthma and reduction in the requirement for regular use of ICS and beta- 2 agonists.

Randomized controlled trials (RCTs) have shown that OD use of an ICS with a fast-acting beta-2 agonist (FABA) without any regular treatments (NON-REG + OD-ICS / FABA) might be an alternative to regular ICS plus OD-FABA (REG-ICS + OD-FABA) in patients with mild asthma ${ }^{4)}$. In these trials, FABA included not only short-acting beta-2 agonists but also formoterol, because formoterol is used for both maintenance and relief of symptoms because of its pharmacokinetic characteristics of fast action and long duration of action.

Another RCT did not demonstrate a significant difference in efficacy for asthma control between NON-REG + OD-ICS / FABA and an OD-SABA without any regular treatment (NON$\mathrm{REG}+\mathrm{OD}-\mathrm{SABA})^{5)}$. An RCT in patients with moderate asthma showed the inferiority of NON-REG + OD-ICS / FABA for asthma control relative to regular ICS with a long-acting beta-2 agonist (LABA) plus OD-SABA (REG-ICS / LABA + OD-SABA ${ }^{6}{ }^{\text {. }}$.

We previously investigated the non-inferiority of NON-REG + OD-ICS/FABA relative to REG-ICS + OD-SABA or REG-ICS / LABA + OD-SABA in patients with mild to moderate asthma in a network meta-analysis of RCTs. Non-inferiority of NON-REG + OD-ICS / FABA to REG-ICS + OD-SABA or REG-ICS / LABA + OD-SABA was not observed, but the probability of being the best treatment was higher for NON-REG + OD-ICS / FABA than for REG-ICS + OD-SABA (unpublished results). This suggests that NON-REG + OD-ICS / FABA may be an alternative therapy to REG-ICS + OD-SABA in patients with mild to moderate asthma, and especially in those with poor adherence to conventional regular treatment. However, the efficacy of NON-REG + OD-ICS / FABA compared to the guideline-recommended conventional treatment is uncertain, and the ideal population of patients who may benefit from the treatment remains undefined. To examine these questions, we conducted a network meta-analysis of RCTs to assess the non-inferiority of NON-REG + OD-ICS / FABA to REG-ICS + OD-SABA in patients with mild asthma.

\section{Methods}

Publication search and inclusion criteria

MEDLINE (source, PubMed), Scopus, and the Cochrane library database (up to August 2016) were searched using the terms "on-demand", “as-needed", "symptom-driven", and "mild asthma". PubMed was mainly used for the publication search because it is an open access database that 
can be used for a comprehensive literature search. Scopus was used to ensure that all eligible articles were detected in PubMed. We also used the Cochrane library database to search for additional references. Embase was not used because it is unavailable in our institute. This is unlikely to have had a significant impact on the search results owing to the similarities between PubMed and Embase. No restrictions were imposed on the search language. A study was considered potentially eligible if it met the following criteria : 1) a RCT that assessed the clinical efficacy of NON-REG + OD-ICS / FABA, REG-ICS + OD-SABA, or NON-REG + OD-SABA in mild asthma; and 2) it included asthma control as an outcome measure.

\section{Data extraction and quality assessment}

Studies in children aged 18 years or younger, non-English-language studies, case reports, and single arm studies were excluded. The methodological quality of the included trials was evaluated using the Jadad score, which assesses studies based on their description of randomization, blinding and dropout ${ }^{7)}$.

\section{Data analysis}

A network meta-analysis was performed to compare the efficacy of NON-REG + OD-ICS / FABA with that of REG-ICS + OD-SABA and NON-REG + OD-SABA for asthma control, using the statistical method described by White ${ }^{8)}$. The summary effect size is expressed as the mean difference (MD). One advantage of a network meta-analysis is that the analysis can be performed even if the assessment of asthma control differs among the included RCTs because the methodological framework is based on the concept of a multivariable meta-analysis ${ }^{8,9)}$. Non-inferiority for the incidence of asthma control was defined as the upper $95 \%$ confidential intervals (CIs) for the MD estimated in the network meta-analysis being no higher than 0.1. Data analysis was performed using STATA ver. 14.0 (Stata Corp., College Station, TX).

\section{Ranking investigation}

The probability of being the best treatment and the ranks of each treatment can be evaluated using statistical methods in a network meta-analysis. Thus, the calculated probability of being the best treatment, second best treatment, third best treatment, etc., can be determined. The surface under the cumulative ranking (SUCRA) curve, which is the ratio of the area under the cumulative ranking curve to the entire area in the plot, compares each treatment to an ideal best treatment, with a larger SUCRA value indicating a more effective treatment ${ }^{8,9)}$.

\section{Inconsistency test}

A network meta-analysis is carried out based on the assumption of consistency, which should be confirmed in a closed loop of evidence. The difference between direct and indirect evidence is expressed as the inconsistency factor, with $95 \%$ CIs and a $P$-value, and is calculated by analyzing the equality of direct and indirect evidence. CIs are truncated to zero because inconsistency factors represent the absolute difference between direct and indirect evidence. Loops in 


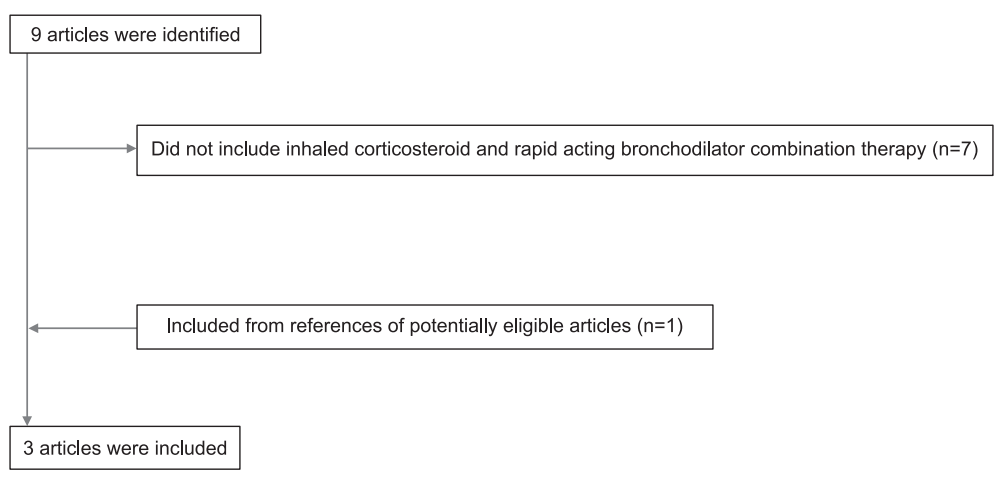

Fig. 1. Flow diagram of the study selection process

which the lower CI limit does not reach the zero line indicate that significant inconsistency is present $^{8,9)}$.

\section{Comparison-adjusted funnel plot}

A comparison-adjusted funnel plot is a scatter plot that reveals the association between studyspecific effect sizes from the corresponding comparison-specific summary versus the inverted standard error. An asymmetrical funnel plot implies the presence of small-study effects, which reflect differences in effectiveness between small and large studies ${ }^{8,9)}$.

\section{Results}

Search results and characteristics of included studies

The study selection process is shown in Figure 1. Nine citations were retrieved from the databases, of which 7 did not examine NON-REG + OD-ICS / FABA. One article was identified from the reference lists of included studies. Finally, three RCTs were included in the network meta-analysis ${ }^{4,5,10)}$. The characteristics of these studies are shown in Table 1. NON-REG + OD-ICS / FABA was included in 2 trials, REG-ICS + OD-SABA in 3 trials, and NON-REG + OD-SABA in 3 trials. All comparisons within the analysis are shown in Figure 2. The mean age ranged from 28 to 45 years and the study duration ranged from 6 weeks to 6 months. The methods of evaluation of asthma differed among the three RCTs: the daytime asthma score was used in 2 RCTs, and the asthma control questionnaire score was used in one. The Jadad scores for the three studies ranged from 3 to 5 , indicating that the included studies were of high quality.

\section{Treatment comparison for asthma control in mild asthma}

The results of a comparison of the efficacy of the treatments for mild asthma control are shown in Table 2. Non-inferiority of NON-REG + OD-ICS / FABA to REG-ICS + OD-SABA was shown because the upper 95\% CI for MD was within the non-inferiority margin (MD, $-0.17 ; 95 \% \mathrm{CI},-0.41$ to 0.07$)$. Superiority of NON-REG + OD-ICS / FABA to NON-REG + OD-SABA was also shown (MD, $-0.29 ; 95 \% \mathrm{CI},-0.49$ to -0.08$)$. 
Treatment for Mild Asthma (25 Characters)

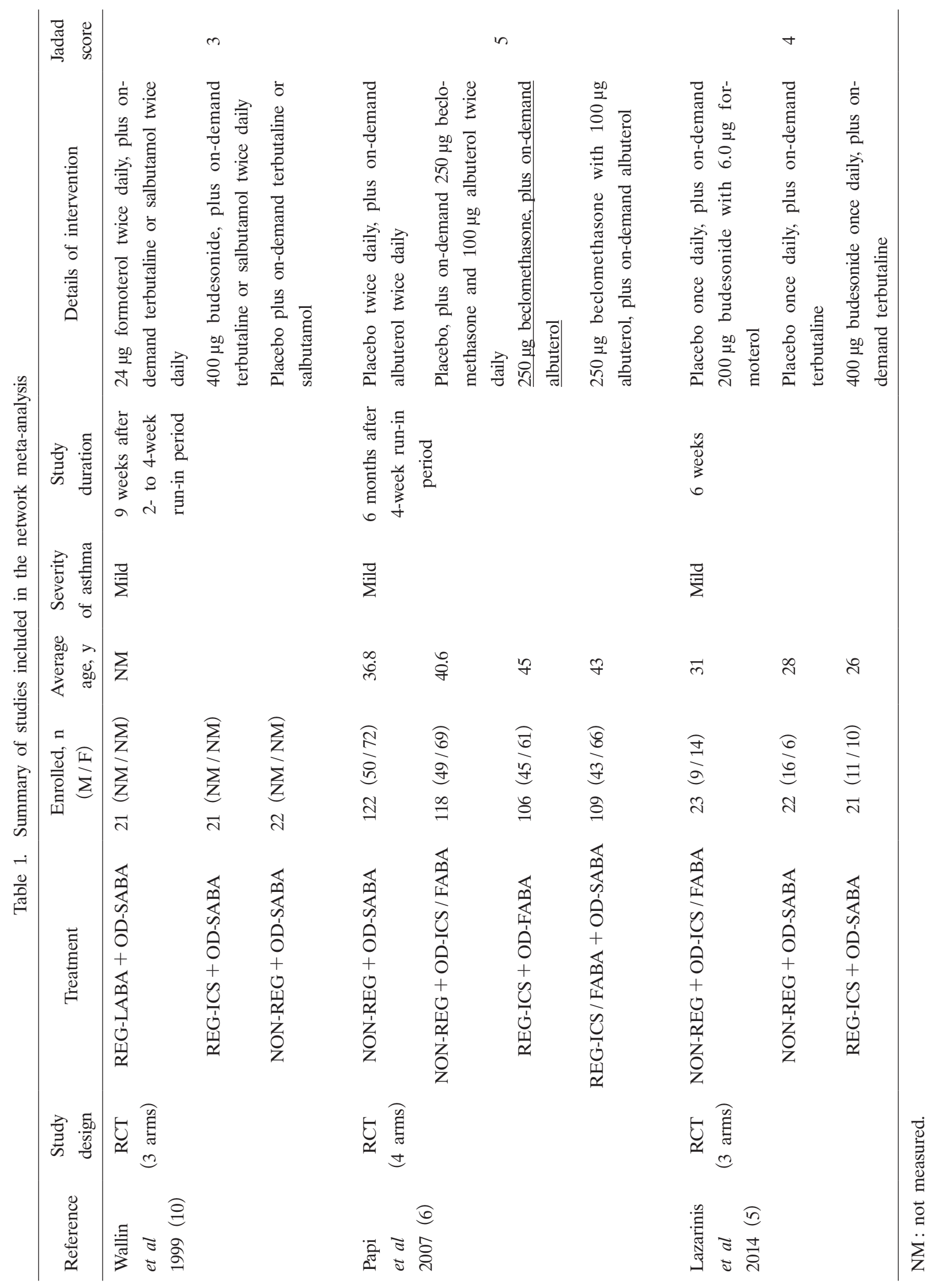




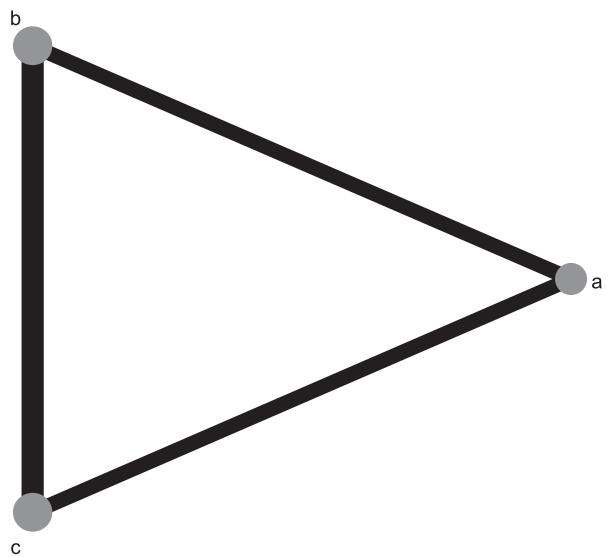

Fig. 2. Network of eligible comparisons for treatment efficacy in the network meta-analysis The width of the lines represents the number of studies compared for each pair of treatments, and the size of balloons represents the total sample size of each treatment.

a: on-demand inhaled corticosteroid and fast-acting beta-2 agonist without any regular treatment (NON-REG + OD-ICS / FABA).

b : regular inhaled corticosteroid plus on-demand short-acting beta-2 agonist (REG-ICS + OD-SABA).

c: on-demand short-acting beta-2 agonist without any regular treatment (NON-REG + OD-SABA).

Table 2. Summary of non-inferiority and superiority of NON-REG + OD-ICS / FABA relative to REG-ICS + OD-SABA and NON-REG + OD-SABA for control of mild asthma

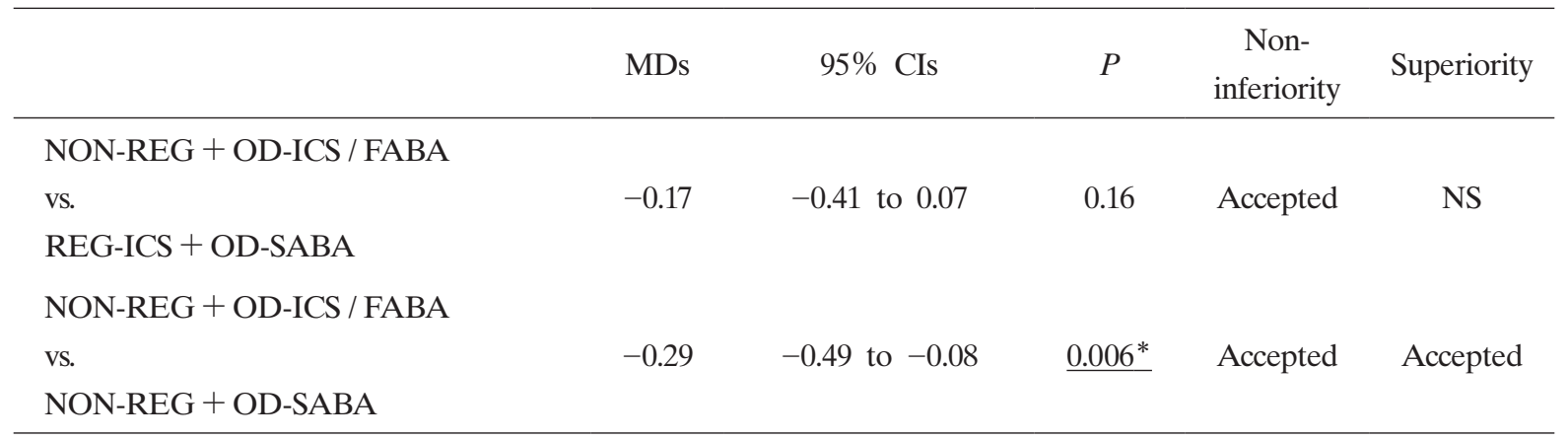

Results are expressed as mean differences (MDs) with 95\% confidence intervals (CIs).

NON-REG + OD-ICS / FABA : on-demand inhaled corticosteroid and fast-acting beta-2 agonist combination without any regular treatment; REG-ICS + OD-SABA : regular inhaled corticosteroid plus on-demand shortacting beta-2 agonist; NON-REG + OD-SABA : on-demand short-acting beta-2 agonist without any regular treatment; NS : not significant.

${ }^{*} P<0.05$ for differences between groups.

\section{Analysis of ranking probability}

The ranks of NON-REG + OD-ICS / FABA, REG-ICS + OD-SABA, and NON-REG + ODSABA are shown in Table 3. The respective probabilities of being the best treatment were $91.2 \%, 8.8 \%$, and $0.2 \%$; those for being the second best treatment were $8.6 \%, 79.0 \%$, and $12.3 \%$; and those for being the third best treatment were $0.2 \%, 12.2 \%$, and $87.6 \%$ (Table 3). The SUCRA values for NON-REG + OD-ICS / FABA, REG-ICS + OD-SABA, and NON-REG 
Table 3. Ranking probabilities and surface under the cumulative ranking (SUCRA) curve values

\begin{tabular}{lrrrrc}
\hline Treatment & Best & $2^{\text {nd }}$ & $3^{\text {rd }}$ & $\begin{array}{c}\text { Mean } \\
\text { rank }\end{array}$ & SUCRA \\
\hline NON-REG + OD-ICS / FABA & 91.2 & 8.6 & 0.2 & 1.1 & 1.0 \\
REG-ICS + OD-SABA & 8.8 & 79.0 & 12.2 & 2.0 & 0.5 \\
NON-REG + OD-SABA & 0.2 & 12.3 & 87.6 & 2.9 & 0.1 \\
\hline
\end{tabular}

Probabilities of being the best, second best, or third best treatment, calculated from the network meta-analysis, and SUCRA values for mild asthma control.

NON-REG + OD-ICS / FABA : on-demand inhaled corticosteroid and fast-acting beta-2 agonist combination without any regular treatment; REG-ICS + OD-SABA : regular inhaled corticosteroid plus on-demand short-acting beta-2 agonist; NON-REG + OD-SABA : on-demand short-acting beta-2 agonist without any regular treatment.

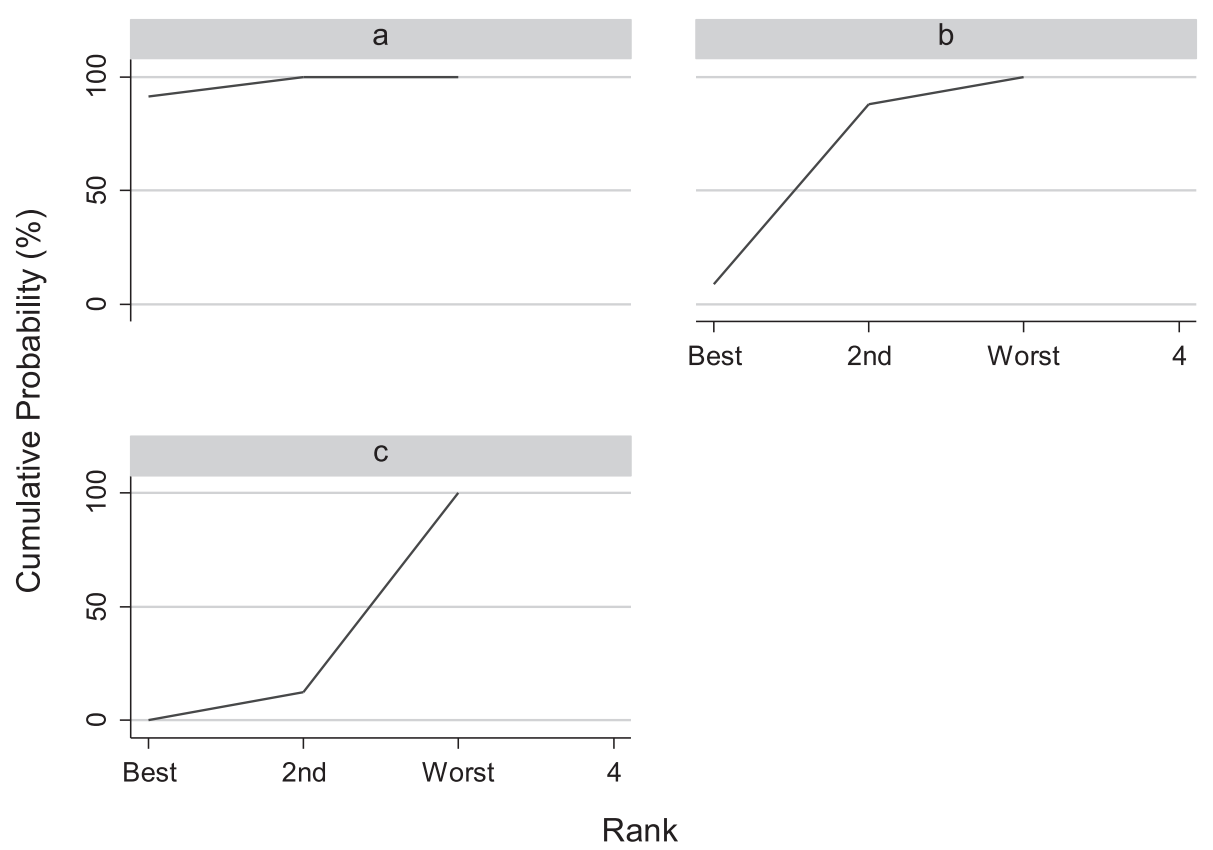

Fig. 3. Surface under the cumulative ranking curves for asthma control

a: on-demand inhaled corticosteroid and fast-acting beta-2 agonist without any regular treatment (NON-REG + OD-ICS / FABA).

$\mathrm{b}$ : regular inhaled corticosteroid plus on-demand short-acting beta- 2 agonist (REGICS + OD-SABA).

c: on-demand short-acting beta-2 agonist without any regular treatment (NON-REG + OD-SABA).

+ OD-SABA for asthma control were 1.0, 0.5, and 0.1, respectively (Fig. 3, Table 3).

\section{Inconsistency test}

A funnel plot (Fig. 4) showed symmetry to the line, which suggests that the network metaanalysis did not include a small-study effect. An inconsistency plot for direct and indirect 


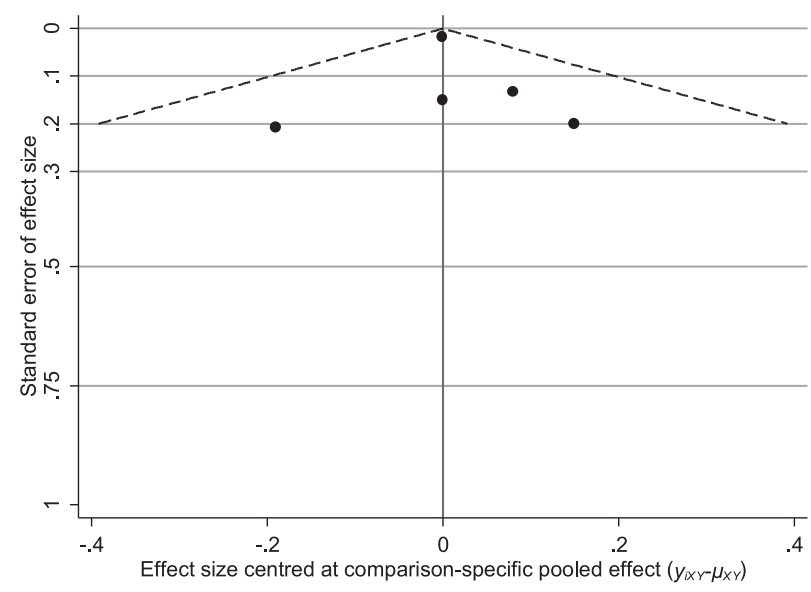

Fig. 4. Comparison-adjusted funnel plot for the network meta-analysis

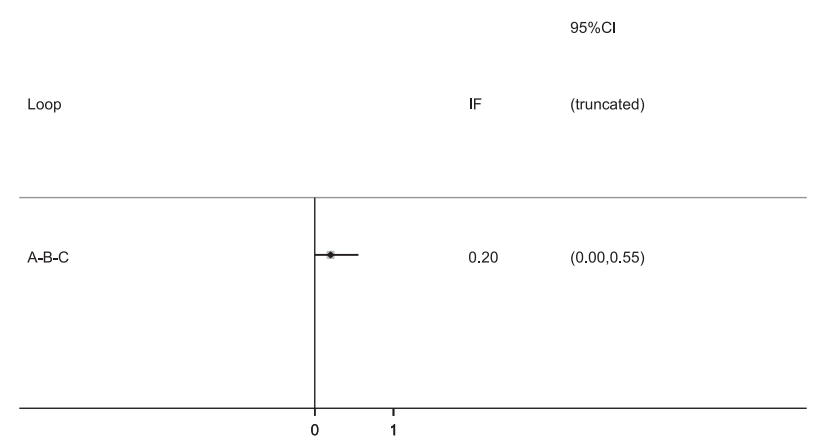

Fig. 5. Inconsistency plot for the network meta-analysis IF : inconsistency factor; CI : confidence interval.

comparisons (Fig. 5) suggested no significant inconsistency because the corresponding CI included zero.

\section{Discussion}

In this network meta-analysis, we showed non-inferiority of NON-REG + OD-ICS / FABA for asthma control relative to REG-ICS + OD-SABA, and superiority of NON-REG + OD-ICS / FABA over NON-REG + OD-SABA in patients with mild asthma. NON-REG + OD-ICS / FABA also had the highest probability of being the best treatment and had the highest SUCRA value among the three treatments.

Previous RCTs have shown mixed results for asthma control after treatment with NON-REG + OD-ICS /FABA, partially because of differences in the distribution of asthma severity and definitions of complications ${ }^{4-6)}$. In this study, we focused on mild asthma, and demonstrated non-inferiority of NON-REG + OD-ICS / FABA to REG-ICS + OD-SABA. As expected, the efficacy of NON-REG + OD-ICS / FABA for asthma control was superior to REG-ICS + ODSABA. This is plausible because an ICS and beta stimulant combination inhibits bronchoconstriction and suppresses airway inflammation.

This is the first network meta-analysis of the efficacy of NON-REG + OD-ICS / FABA focusing on mild asthma and this treatment was the highest ranked among the three treatments. We previously assessed non-inferiority of NON-REG + OD-ICS / FABA to conventional best practice for asthma control or incidence of asthma exacerbation or aggravation in mild to moderate asthma (not published). The results did not show non-inferiority of NON-REG + OD-ICS / FABA to REG-ICS + OD-SABA and REG-ICS / LABA + OD-SABA. Taken together, those results and the current results suggest that NON-REG + OD-ICS / FABA is effective as an alternative to the guideline-recommended conventional treatment in patients with mild asthma, rather than mild to moderate asthma, and especially in patients whose adherence to regular treatment is low.

Several limitations of this study should be acknowledged. First, only three articles were 
included in the analysis. Second, a meta-analysis is a form of retrospective research that is subject to the metrological deficiencies of the studies included in the analysis. Finally, only published studies were included, and some publication bias may thus be present.

In summary, the non-inferiority of NON-REG + OD-ICS / FABA to REG-ICS + OD-SABA and the superiority of NON-REG + OD-ICS/FABA to NON-REG + OD-SABA were demonstrated in this study. Moreover, NON-REG + OD-ICS / FABA was ranked as the best treatment among the three approaches. These results suggest that NON-REG + OD-ICS/FABA may be beneficial as an alternative therapy to REG-ICS + OD-SABA in patients with mild asthma, and particularly in patients with low adherence to regular conventional treatment. Considering the limitations of this meta-analysis, there is a need for further research to confirm the efficacy of treatment using NON-REG + OD-ICS / FABA for mild asthma.

\section{Conflict of interest disclosure}

The authors have declared no conflict of interest.

\section{References}

1) Corre KA, Rothstein RJ. Assessing severity of adult asthma and need for hospitalization. Ann Emerg Med. 1985;14:45-52.

2) Ohta K, Ichinose M, Nagase H, et al. Japanese guideline for adult asthma 2014. Allergol Int. 2014;63:293-333.

3) Weinstein AG. Selecting the correct treatment for asthma adherence studies. J Allergy Clin Immunol. 2016;137:1916.

4) Papi A, Canonica GW, Maestrelli P, et al. Rescue use of beclomethasone and albuterol in a single inhaler for mild asthma. $N$ Engl J Med. 2007;356:2040-2052.

5) Lazarinis $\mathrm{N}$, Jorgensen L, Ekstrom $\mathrm{T}$, et al. Combination of budesonide/formoterol on demand improves asthma control by reducing exercise-induced bronchoconstriction. Thorax. 2014;69:130-136.

6) Papi A, Marku B, Scichilone N, et al. Regular versus as-needed budesonide and formoterol combination treatment for moderate asthma: a non-inferiority, randomised, double-blind clinical trial. Lancet Respir Med. 2015;3:109-119.

7) Jadad AR, Moore RA, Carroll D, et al. Assessing the quality of reports of randomized clinical trials: is blinding necessary? Control Clin Trials. 1996;17:1-12.

8) White IR. Network meta-analysis. Stata J. 2015;15:951-985.

9) Chaimani A, Higgins JP, Mavridis D, et al. Graphical tools for network meta-analysis in STATA. PLoS One (Internet). 2013;8:e76654. (accessed 2016 Sep 1) Available from: http://journals.plos.org/plosone/article?id=10.1371/ journal.pone.0076654

10) Wallin A, Sandstrom T, Soderberg M, et al. The effects of regular inhaled formoterol, budesonide, and placebo on mucosal inflammation and clinical indices in mild asthma. Am J Respir Crit Care Med. 1999;159:79-86.

[Received September 29, 2016 : Accepted October 14, 2016] 